\title{
FORMACIÓN EN INVESTIGACIÓN DE DOCENTES DE ENFERMERÍA DE LA UNIVERSIDAD NACIONAL AUTÓNOMA DE MÉXICO (UNAM)
}

UNIVERSITY NURSING STAFF FORMATION IN RESEARCH AT UNAM'S

Recibido 5 de abril de 2017 Aceptado 14 de julio de 2017
Palabras clave: docente, enfermería universitaria, formación en investigación, recursos humanos.

Key words: Professor, University Nursing, Formation in Research, Human Resources.

\section{Autores:}

Dra. Lasty Balseiro Almario

Responsable del Proyecto. Prof. de Carrera Titular "B". Adscrita a la Unidad de investigación de la ENEOUNAM Cd. de México Correo: lastybals@hotmail.com,

Dra. Gandhy Ponce Gómez

Coordinadora de la Maestría en Enfermería de la UNAM. Prof. de Carrera Titular "A" de la ENEO Cd. de México

Mtra. Margarita Acevedo Peña

Coordinadora de la Carrera de Enfermería FES Iztacala UNAM

Dra. Silvia Crespo Knopfler

Decana de la Carrera de Enfermería. FES Zaragoza. Prof. de Carrera Titular "B" de la FES Zaragoza UNAM.

Dra. Ma. Susana. González Velázquez Coordinadora de la Carrera de Enfermería FES Zaragoza UNAM

Mtro. Adiel Agama Sarabia

Prof. de Carrera Asociado "C" de la FES Zaragoza UNAM

PSTE. Giordano Sandro Vázquez Lira PSTE. Daniela Javier Cabrera

Pasantes de la Licenciatura de Enfermería y Obstetricia ENEO-UNAM.

60 
RESUMEN

Objetivo: El objeto de esta investigación fue diagnosticar el estatus de la formación en investigación de los docentes de Enfermería de la UNAM.

Metodología: El diseño metodológico fue de corte cuantitativo, no experimental, transversal, descriptivo y diagnóstico. En la primera fase se plantea un diagnóstico situacional de la formación de docentes en investigación, en la segunda, iniciar una intervención educativa en materia de investigación y en la tercera, evaluar el impacto de la intervención educativa. Este reporte da cuenta de la primera fase de la investigación.

Resultados: De los 44 docentes encuestados, el 35\% son Titulares A, B, C, el $73 \%$ Asociados A, B, C, el 54\% de Asignatura $A$ y $B$, el 25\% tienen grado de Doctorado, el 43.10\% Maestría y el 17.40\% Especialidad de Posgrado. En cuanto a la formación en investigación el $47 \%$ de los docentes "a veces" ha tomado cursos de teoría del conocimiento, el $40.9 \%$ "a veces" ha tomado cursos de metodología de la investigación, el 53.70\% "nunca" ha tomado seminarios de artículos de revisión y el $47.60 \%$ nunca han tomado cursos para elaborar ensayos.

Conclusiones: La formación en investigación de los docentes está en los rangos de "a veces y muy pocas veces". Esto da idea de que es escasa la formación en investigación en la Escuela Nacional de Enfermería y Obstetricia (ENEO), la Facultad de Estudios Superiores (FES) Iztacala y la Facultad de Estudios Superiores (FES) Zaragoza de la UNAM y que es necesario incentivar la formación de investigación.

Palabras clave: docente, enfermería universitaria, formación en investigación, recursos humanos.
ABSTRACT

Objective: To assess the research formation status of the university nursing professors at UNAM's.

Methodology: This study used a quantitative, nonexperimental, transversal, descriptive, and diagnostic methodological design. In the first phase, a situational diagnosis of the research professors is presented. In the second phase, a research-related educational intervention is carried out. In the third phase, the educational intervention is evaluated. This report shows the first phase of the research.

Results: From the 44 professors surveyed, 35\% are Career Professors "A", "B", or "C"; $13 \%$ are Associated Professors " $A$ ", "B", or " $\mathrm{C}$ "; and 54\% are Subject Professors "A" or "B". Twenty five percent have a PhD; $43.10 \%$ have a Masters Degree; and 11.40\% have a Graduate Specialty. In relation to the professors' formation in research, $47 \%$ have "sometimes" taken knowledge theory courses; 40.9\% have "sometimes" taken research methodology courses; $50.70 \%$ have "never" taken review articles seminaries; and $47.60 \%$ have "never" taken courses in essay-writing.

Conclusions: The research formation among these professors lies within the ranges of "some to very few times" in relation to their research courses taking. This suggests that there is a lack of research training in the three Entities and that it is necessary to encourage research training.

Key words: Professor, University Nursing, Formation in Research, Human Resources. 


\section{INTRODUCCIÓN}

Problema: El problema planteado para este estudio fue conocer el estatus en la formación en investigación de los docentes de la Enfermería de la Universidad Nacional Autónoma de México (UNAM).

Marco Teórico: Formar docentes de enfermería en investigación, requiere como estrategia el aprendizaje de conocimientos teóricos, metodológicos y técnicos del quehacer científico, que les permita desarrollar destrezas, actitudes y conductas investigativas. Por ello, para Zarza Ma. D?' uno de los aspectos más importantes que garantiza la productividad científica en enfermería es la formación de recursos humanos en investigación. Así, en la Enfermería Universitaria: Escuela Nacional de Enfermería y Obstetricia (ENEO), la Facultad de Estudios Superiores (FES) Iztacala y Facultad de Estudios Superiores (FES) Zaragoza de la UNAM, se han realizado acciones que permiten el incremento, por una parte, del número de estudiantes de pregrado y posgrado en proyectos de investigación como becarios o pasantes de servicio social, de manera tal que no solo aprendan investigación con los docentes, sino que obtengan grados académicos por las diversas modalidades aprobadas por la UNAM, contribuyendo con ello a la productividad de los docentes con quienes estos alumnos se incorporan.

Por otra parte, para Harrison L y Cols. ${ }^{2}$, la formación de docentes es esencial para desarrollar, evaluar y expandir el conocimiento de enfermería. Así, la capacitación de docentes, según Miotto M.G y Garzón $N^{3}$ es tan necesaria que los gobiernos y las universidades deben promover en cada país, de acuerdo con las políticas de ciencia y tecnología en vigor, la creación de Centros de Investigación que integren la enseñanza, la práctica, y la investigacion a fin de buscar soluciones a los problemas que enfrentan los servicios de salud y la profesión de Enfermería. Por lo anterior, el interés de este estudio es diagnosticar el estatus de la formación en investigación de los docentes de la Enfermería Universitaria: ENEO, FES Iztacala y FES Zaragoza de la UNAM, para después, fortalecer la capacitación de los académicos con las distintas metodologías de la investigación. Este fortalecimiento implica adquirir mayores aptitudes investigativas con: cursos de Teoría del Conocimiento, Método Científico, Metodología de la Investigación, Estadística descriptiva y Estadística inferencial, Investigación Cuantitativa y Cualitativa. Conviene también la implementación de talleres para la Elaboración de capitulos de libros y, Búsqueda bibliográfica y la realización de Seminarios para la redacción de artículos de revisión y Elaboración de ensayos, entre otros.

Unejemplo importante sobre la eficacia de programas de capacitación en investigación se observa en los resultados obtenidos por Mercado F.J y Cols. ${ }^{4}$ con la investigación de corte cuantitativo realizada en una Facultad de Enfermería de la UNAM en la que se comprueba el fortalecimiento de la investigación a partir de la participación de 16 docentes, investigadores y alumnos de Licenciatura y Posgrado a los cursos de Metodología de la investigación, seminarios de investigación, cursos, talleres y asesorías individualizadas en el quehacer investigativo. Esta iniciativa denominada Plataforma de Investigación se basó en principios de colaboración y participación de los actores involucrados en donde grupos académicos de investigadores apoyan, de manera presencial y/o virtual, con seminarios especializados de metodología de la investigación, los avances de los proyectos, los borradores de sus artículos y los ensayos científicos. En los resultados, los docentes mostraron diversas habilidades investigativas, adquirieron conocimientos y sometieron sus proyectos a concursos de organismos estatales y nacionales.

De igual forma, en otra investigación realizada por Cisneros M. ${ }^{5}$ del Instituto de la Amazonia Peruana, se conjuntó una experiencia de formación docente revisando los recursos metodológicos para mejorar la redacción de artículos científicos y de revisión y con ello, presentar mejores manuscritos en la Revista Científica Folia Amazónica y lograr su indización en bases internacionales. Los cursos y talleres fueron teórico práctico y recorrieron cuatro etapas: la elaboración de un proyecto, la ejecución de la investigación, la redacción de un informe

\footnotetext{
62 IMN Nhy Enf Neurol Vol.16. No. 2 mayo - agosto 2017
} 
final y la publicación de sus resultados en un artículo científico. Así, en el Instituto de la Amazonia Peruana, después de la intervención educativa, el 63\% de los profesores involucrados dijeron que mejoraron notablemente en los aspectos básicos de la información y redacción del artículo o ensayos publicados. Entonces, a partir del desarrollo de estas habilidades, se crearon los grupos de investigación, redes locales y regionales que, bajo el liderazgo de investigadores reconocidos, impulsan el desarrollo de los grupos de profesores que apenas están incursionando en publicar.

De la misma manera, en una investigación de Faccia $K^{6}$, de corte cuantitativo y cualitativo con triangulación metodológica y con una muestra de 688 alumnos de enfermería y 20 profesores de salud y enfermería, en la que se busca conocer las representaciones sociales de la profesión y la formación desde una perspectiva antropológica de enfermería en Buenos Aires, se encontró que se requiere fortalecer las políticas de capacitación de recursos humanos para mejorar las condiciones de formación y de trabajo. Las conclusiones plantean que para poder producir trasformaciones en la atención de enfermería se requiere un trabajo mancomunado entre el servicio, la docencia y las asociaciones profesionales. Para ello, es necesario establecer estrategias que conduzcan a la incorporación de la fundamentación científica, de la teoría y de la práctica profesional para una sólida formación política, disciplinar, pedagógica, científica, humanística, ética y estética, ya que solo asi se podrá atender mejor quienes estén al cuidado de las enfermeras.

En el caso de Brasil, para Palucci M.H.?, el proceso de producción del conocimiento siempre estuvo vinculado al crecimiento de los cursos de postgrado. Así, el país busca, principalmente por medio de estos cursos, consolidar su base científica y formar recursos humanos capacitados para solucionar problemas regionales y nacionales. Un objetivo importante del sistema de formación es preparar investigadores que puedan cumplir tales objetivos. Esos productores del conocimiento deben poseer dominio del estado de conocimiento en su área de actuación y capacidad de organizar preguntas coherentes y actualizadas con dominio metodológico para testarlas. Así, con la estructuración de líneas de investigación autóctonas, se vuelven multiplicadores en la formación de nuevos investigadores y la producción originada debe ser refrendada por evaluadores externos del contexto nacional e internacional.

Por ello, según Lorenzini A. y Dumet J.8, el Posgrado de la Enfermería Brasileña se está consolidando en la formación de recursos humanos en investigación formando Maestros en Ciencias de la Enfermería desde los años 70's y Doctores de enfermería desde los 80 's. Para ello, cuentan con 35 programas de Posgrado de los cuales hay 17 cursos doctorales, 32 de maestría y 330 grupos de investigación. Su consolidación se da también a través de su internacionalización, por la visibilidad e inserción internacional, a partir de las fortalezas regionales y liderazgos en Sudamérica. Así, la producción del conocimiento avanza apoyada por la visión de la interdisciplinaridad, intersectorialidad y para incrementar el número de publicación de alto impacto, absorbidas e incorporadas en la práctica y disponibles en revistas científicas cada vez más calificadas.

Lo anterior se pone de manifiesto en la investigación realizada por Díaz L.P y Cols. ${ }^{9}$ en Sao Paolo, Brasil, para identificar las características y las particularidades publicadas en dos revistas Latinoamericanas en dos países que cuentan con programas de formación a nivel doctoral y que tuviesen una publicación seriada como base de socialización de los resultados. En este estudio, se encontró que las enfermeras que publican son las que tienen formación docente con Maestría y Doctorado. Por el contrario, en las enfermeras asistenciales y estudiantes de pregrado de enfermería, sus publicaciones son de nivel menor. Por lo anterior, es posible que una motivación de los docentes sea dar cumplimiento a un requisito académico y de interés institucional, pero con el predominio del abordaje cuantitativo en las investigaciones, señala la necesidad de incorporar 
nuevos paradigmas interpretativos para responder de mejor manera a las necesidades poblacionales. Por otro lado, es evidente que son los docentes quienes más investigan. Esto se da porque existe la necesidad de mayor formación y motivación para la investigación, especialmente ahora que la tecnología, la comunicación y la evidencia son parte importante de la sensibilización.

Así, la necesidad de formar docentesen investigación, también se pone de manifiesto en el Instituto de Salud Carlos III de España. ${ }^{10}$, para promover la investigación en enfermería ya que, es necesaria esta formación así como buscar estrategias y recursos que posibiliten a los profesionales de enfermería llevar a cabo tareas de investigación y aplicación de los resultados. El desarrollo significa planear la capacitación para efectuar los cambios en los logros de la investigación. De hecho, las experiencias docentes relacionadas con la investigación deberán ser introducidas de manera progresiva desde la educación básica, hasta los niveles superiores, debiendo tomar medidas para preparar un número de profesionales con grado de doctor. La preparación educativa para la investigación también debiera tener lugar en instituciones de enseñanza superior, para lo cual es necesario que la preparación para la investigación no se dé de forma aislada, debiendo incluirse en un marco de referencia científica de enfermería. En una fase temprana de desarrollo, por ejemplo, podría ser necesario realizar acuerdos de cooperación con otras universidades experimentadas en el área de investigación.

\section{METODOLOGÍA}

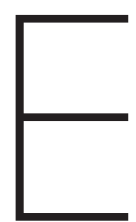

studio de carácter cuantitativo, no experimental, longitudinal, prolectivo, descriptivo y diagnóstico, con pretest, cuyo propósito es evaluar una intervención educativa para fomentar y fortalecer a los académicos de la ENEO y sus pares: FES Iztacala y FES Zaragoza en materia de investigación y mejorar la publicación científica de la enfermería universitaria. La variable medida fue Formación de docentes en investigación, con 12 indicadores que son: tomar cursos de teoría del conocimiento, metodología de la investigación, estadística descriptiva e inferencial, investigación cualitativa y cuantitativa; talleres de elaboración de libros y de búsquedas bibliográficas; seminarios de elaboración de artículos de revisión, de artículos originales, de ensayos y de ensayos científicos. Además de su participación en la actualización de planes y programas de estudio.

El estudio se lleva a cabo en 3 etapas: en la primera se hizo un análisis del estado del arte de la capacitación de docentes en investigación en el ámbito Latinoamericano y Mexicano. En la segunda etapa iniciará, derivado del diagnóstico, la intervención educativa con formación en investigación por semestres para la elaboración de diseños de investigación, redacción del escrito científico, estrategias para la publicación, elaboración de libros, redacción de artículos de revisión, elaboración de ensayos científicos. para que los académicos involucrados puedan desarrollar la publicación científica en sus variadas formas. En la tercera etapa, se evaluará el impacto de la intervención educativa. Este reporte da cuenta de la primera etapa, es decir, del pretest.

La población estudiada fue de 81 docentes y, para la selección de la muestra, se consideraron 44 cuya figura y perfil académico permite la productividad en investigación como parte de sus funciones. Es decir, Profesores de Carrera y Asociados A, B y C y Titulares A, B y C. Se incluyen en esta muestra, 38\% de docentes de la ENEO 32\% de la FES Iztacala y el 30\% de la FES Zaragoza. También se incluyen profesores que desean la formación docente en investigación, es decir, profesores de Asignatura A y B que colaboran con los investigadores. Se elaboró un instrumento con preguntas cerradas-estructuradas a partir de los indicadores establecidos, para detectar las necesidades de capacitación de los docentes y fué aplicado durante los primeros 3 meses del 2015.

El procesamiento de los datos se realizó con el paquete de estadísticas para las Ciencias Sociales SPSS. En cuanto a los aspectos éticos, se tomó en cuenta el Reglamento de la Ley General de Salud, de México, especialmente en el título II capítulo 1, artículo 14, fracción I, en los cuales se hace

\section{4} LMN/Why Enf Neurol Vol. 16. No. 2 mayo - agosto 2017 
referencia a los principios científicos y éticos en una investigación. De igual forma, el principio de la privacidad consignado en el artículo 16, que señala lo concerniente al anonimato y la confidencialidad de los datos, los cuales son estrictamente de orden académico y científico. Por lo anterior y de conformidad a lo establecido en los artículos 17 y 23 del citado Reglamento, ésta investigación se considera sin riesgo ya que no afecta en ningún sentido a las personas y prevalece el criterio de respeto a la dignidad y la protección de los derechos y bienestar de los seres humanos. Una vez que se les dio a conocer los objetivos de la misma, se respetó la decisión de los docentes de participar o no en ella. El aspecto financiero fue cubierto por el propio grupo investigador.

\section{RESULTADOS}

巨 n las características sociodemográficas de la muestra de estudio se obtuvo que de los 44 docentes entrevistados, el 70.50\% son mujeres. En cuanto a la edad el 38.60\% tienen 51 años o más, el 36.40\% entre 41 y 50 años, el 15.90\% entre 31 y 40 años y $9.10 \%$ es menor a los 30 años. En relación a la categoría laboral el 54.5\% son Profesores de Asignatura A y B, el 25.00\% Profesores de Carrera Titulares "A, B y C", el 13.70\% son Profesores de Carrera Asociados A, B o C y el 6.8\% corresponde a Técnicos Académicos (gráfica № 1 ). En relación a la entidad dónde laboran los docentes el 38.10\% son de la ENEO, el $31 \%$ de la FES Iztacala, el 28.60\%, FES Zaragoza y un profesor (2.4\%) labora fuera de las tres entidades participantes.

En cuanto al turno de trabajo, el 46.50\% labora en el matutino, el $34.90 \%$ son de tiempo completo, y el $16.30 \%$ son del turno vespertino. Con respecto al grado académico, el 43.20\% tienen Maestría, 25\% tienen Doctorado, 20.50\% licenciatura y $11.40 \%$ Especialidad (gráfica N²).

En relación con la formación de los docentes en investigación: el 40.90\% refieren "siempre" haber tomado un curso de metodología de la investigación, el 31.80\% "muy pocas veces" han tomado un curso de este tipo, el 22.70\% "a veces" haberlo tomado.
Sobre cursos de teoría del conocimiento, el 15.9\% manifestó "siempre" tomarlos, mientras que el 47.70\% los ha tomado "algunas veces", el 27.30\% "muy pocas veces" y el 9.10\% "nunca" ha tomado un curso de este tipo. Los talleres de elaboración de libros y artículos son los que presentan menor asistencia ya que el $54.80 \%$ y el $53.70 \%$, respectivamente, refieren "nunca" haber tomado un curso sobre éstas temáticas, el $31 \%$ mencionó que "a veces" han tomado cursos sobre redacción de libros, el 22\% menciono "muy pocas veces" asistieron a cursos de redacción de artículos y el 19.50\% refiere haberlos tomado "algunas veces" y únicamente el $4.80 \%$ y el $4.90 \%$ contestó "siempre" haberlos tomado en ambos casos (gráfica №3).

En cuanto a la asistencia a cursos para elaborar Ensayos científicos, el 47.60\% "nunca" ha asistido a tomarlos, el 23.80\% dicen que los hatomado "a veces" o "muy pocas veces", mientras que sólo el 4.80\% mencionó "siempre" haberlos tomado. En relación a los Seminarios de búsquedas bibliográficas el 42.90\% refirió "a veces" tomarlos, el 28.60\% "muy pocas veces" los ha tomado y 16.70\% "nunca" los ha tomado. En la asistencia a cursos de Estadística Descriptiva, el 33.30\% manifestó "a veces" haberlos tomado, el 23.80\% siempre los ha tomado y el $21.40 \%$ los ha tomado "muy pocas veces" y con el mismo porcentaje, se encuentran los que nunca los han tomado. En contraparte, los cursos de estadística inferencial, el 35.70\% refieren "nunca" haberlos tomado, $31 \%$ "muy pocas veces" los ha tomado, el 19\% "algunas veces" y sólo el 14.30\% refiere haberlos tomado "siempre" (gráfica N4).

En la asistencia a cursos de investigación cuantitativa y cualitativa el $35.70 \%$ y $36.40 \%$ respectivamente, refieren haberlos tomado "a veces", el 23.80\% haber tomado "siempre" cursos de investigación cuantitativa, el 21.40\% refiere "nunca" haber tomado cursos de este tipo y el 19\% mencionó que "muy pocas veces" lo hace. En el caso de la investigación cualitativa el 27.30\% refirió haberlos tomado "muy pocas veces", seguido del 25\% que "nunca" los ha tomado y únicamente el 11.40\% manifestó haber asistido "siempre" a un curso sobre la temática (gráfica N5).

Enf Neurol Vol. 16. No. 2 mayo - agosto 2017 . Wh 
Por último, en cuanto a la participación en el diseño o actualización de planes y/o programas docentes el 33.30\% consideran que han participado "muy pocas veces", el 26.20\% "siempre" lo ha hecho, mientras que el 27.40\% ha participado "la mayoría de las veces" y el 19\% refirió "nunca" haber participado.

\section{DISCUSIÓN}

巨 n relación con la edad de los entrevistados el 15.90\% de los docentes son personas menores de 40 años, es decirjóvenes en plena formación docente, y con evidentes necesidades de capacitación en investigación. Estos resultados contrastan con la investigación de Guerra M.D y Cols." sobre la formación del profesorado universitario nóvel en la Universidad de Sevilla, en donde se encontraron problemas y necesidades en la formación del profesorado para adecuar su perfil docente a nuevos roles y estrategias de enseñanza-aprendizaje. Este programa de formación del profesorado nóvel sirvió no solo para identificar problemas relacionados en la docencia, intercambiar impresiones e inquietudes del quehacer docente, sino también para proponer estrategias de mejora e innovación docente en investigación.

En cuanto a la categoría laboral de los docentes, el 25\% fueron profesores de Carrera Asociados A, B o C, el 25\% profesores de Carrera Titulares A, B y C y el 54.50\% profesores de Asignatura A y B, lo que es similar a la investigación, realizada por Liceras A. ${ }^{22}$, sobre la formación del profesorado en didáctica de las Ciencias Sociales en donde se plantea el reto de formar a 176 profesores universitarios para que estén preparados para hacer frente a las demandas sociales de formación, ayudándoles a resolver sus problemas de aula y a innovar en la metodología de las Ciencias Sociales. Entre los problemas detectados estála falta de infraestructura con carencia de medios y recursos que pudieran dirigir o responsabilizarse de las investigaciones, heterogeneidad de líneas y formas de hacer investigación así como ausencia de equipos estables y consolidados para abordar y desarrollar trabajos y líneas de investigación.
En cuanto a la formación de docentes en investigación, el 40.90\% de los docentes refieren que siempre han tomado cursos de metodología de la investigación y el 31.80\% muy pocas veces; estos resultados se asemejan a la investigación de corte cualitativo realizada por Carro L.13 sobre la formación del profesorado en investigación Educativa en Madrid, España, en donde se evidencia la preocupación constante de los Centros Educativos por mantener actualizada la oferta informativa del profesorado dado que solo el 8\% realiza proyectos de innovación, aunque el 82\% señaló no haber recibido ninguna formación en este campo. Por ello, es necesario formar a los docentes en esta materia y satisfacer las necesidades profesionales de los académicos con las diferentes estrategias de investigación para el desarrollo profesional.

De igual forma, en otra investigación de corte cuantitativo realizada por Guisasola J y Cols. ${ }^{14}$ sobre la formación continua del profesorado, la investigación educativa e Innovación de la enseñanza en la Universidad de Zaragoza, España, se encontró que la formación continua del docente, trata de hacer frente a las deficiencias que ellos tienen sobre las dinámicas de rutina y expectativas negativas de su propia tarea. También se encontró que la formación continua del profesorado es un proceso largo, que implica actualización, desarrollo y puesta en marcha de actividades innovadoras con evaluación de resultados durante todas las etapas en que el profesorado se encuentre en activo.

En cuanto a la participación en diseños o actualización de planes y programas docentes, el 33\% de los académicos de la Enfermería Universitaria, manifiestan que han participado "muy pocas veces", lo que contrasta con la investigación de Romo A.F. y Cols. ${ }^{15}$ relativa a estrategias para la formación de Investigación en la Universidad Jesuita, en Guadalajara, México, en donde se pudo identificar que hasta el año 2007, en esta Universidad el término Proyectos de Investigación no formaba parte de la simbología, lenguaje y conocimiento o innovación de los docentes de la Institución. Por ello, mediante

\section{6}

MMN Why Enf Neurol Vol.16. No. 2 mayo - agosto 2017 
un diagnóstico inicial, se determinan estrategias de formación de los docentes, diseño y desarrollo para la planeación, programación y desarrollo de talleres, seminarios, encuentros académicos, integración de grupos de investigación, apoyo metodológico en proyectos y publicaciones, para posteriormente, hacer las evaluaciones y seguimiento de resultados y hacer que estos docentes ingresen a programas de estímulos para la investigación. Con estas estrategias los docentes han consolidado sus conocimientos generando diversos productos de investigación.

\section{CONCLUSIONES}

$\mid$ ablar deformación docenteen investigación hace referencia no únicamente al desarrollo de un conjunto de conocimientos, teorías, técnicas y metodologías, ofertados a través de programas académicos de Maestrías y Doctorados. Se refiere más bien a la formación en el ejercicio de la investigación como "un oficio", que implica el aprendizaje de ciertos conocimientos teórico-metodológicos y técnicos del quehacer científico en el campo, el desarrollo de destrezas, actividades y conductas investigativas, para conformar el saber y el hacer cotidiano de los profesores, mediante estrategias de formación individuales, grupales e institucionales.

La formación de docentes en investigación de la ENEO, la FES Zaragoza y la FES Iztacala, evidencía insuficiencias que obligan a fundamentar una reconceptualización de la Gestión formadora de los docentes en investigación de la Enfermería Universitaria. Si bien el diagnóstico realizado muestra que falta formación en Investigación de los docentes en las tres instancias de la Enfermería Universitaria también da idea de que es necesario incentivar la formación en investigación para fortalecer sus conocimientos que garanticen la productividad científica porque incluso, los grados académicos de los docentes, no garantizan la solidez y los conocimientos en el campo de la investigación. Una estrategia desarrollada ha sido la creación de la Red Académica y de Investigación en apoyo a la publicación científica de Enfermería en donde los docentes podrían verse beneficiados con cursos, talleres, seminarios de investigación que les garantizan los conocimientos teorico-practicos en esta materia. Este planteamiento conduce a pensar que la formación docente juega un papel relevante en la transformación de la práctica pedagógica, la cual debiera ser integral, colectiva, reflexiva, participativa, investigativa y sistematizada.

Ahora, si se trata de docentes nóveles, aunque ellos no han transitado por la "carrera docente", dado que su función didáctica ha sido adquirida en la práctica, también debe realizarse un esfuerzo adicional, centrado en la formación de quehacer científico, para crear "semilleros de investigación" que más adelante se conviertan en docentes investigadores.

Por lo anterior, los moderados avances en materia de formación de docentes en investigación que han sido evidenciados, requieren una atención inmediata con estrategias y acciones de la Universidad, de los grupos consolidados de investigación, de las redes de investigaciones nacionales e internacionales, encaminados a facilitar el acceso y la apropiación de técnicas, métodos y desarrollo de habilidades, hábitos y actitudes que demanda la práctica investigativa. De esta manera, el docente de la Enfermería Universitaria podrá innovar en el aula y producir conocimiento en relación a su objeto de trabajo. 
Gráfica I. Categoría de los docentes

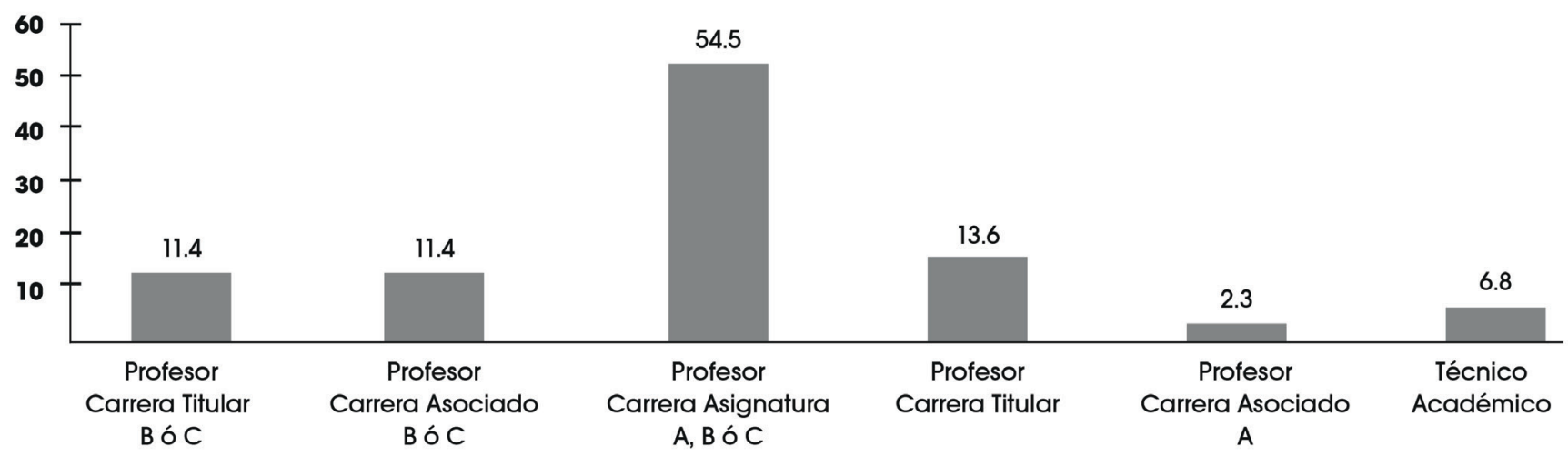

Gráfica 2. Grado académico de los docentes

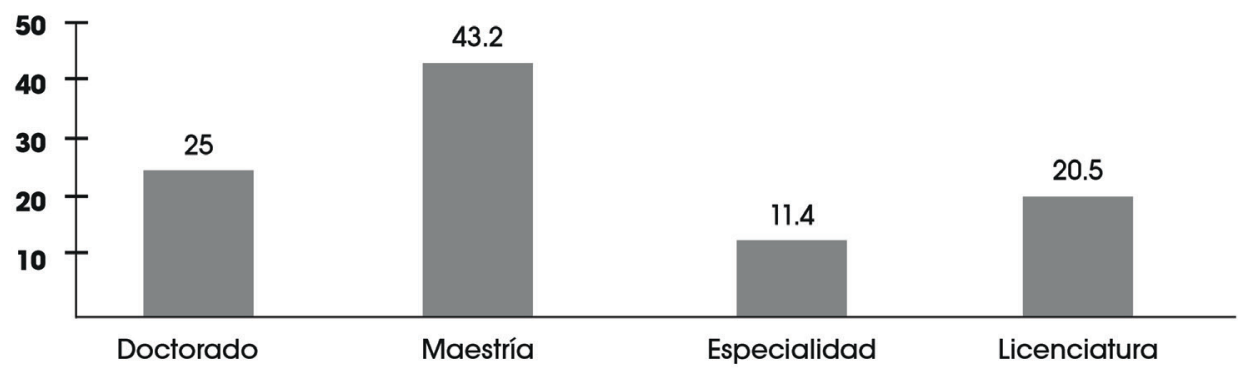

Gráfica 3. Asistencia de docentes a cursos, talleres o seminarios

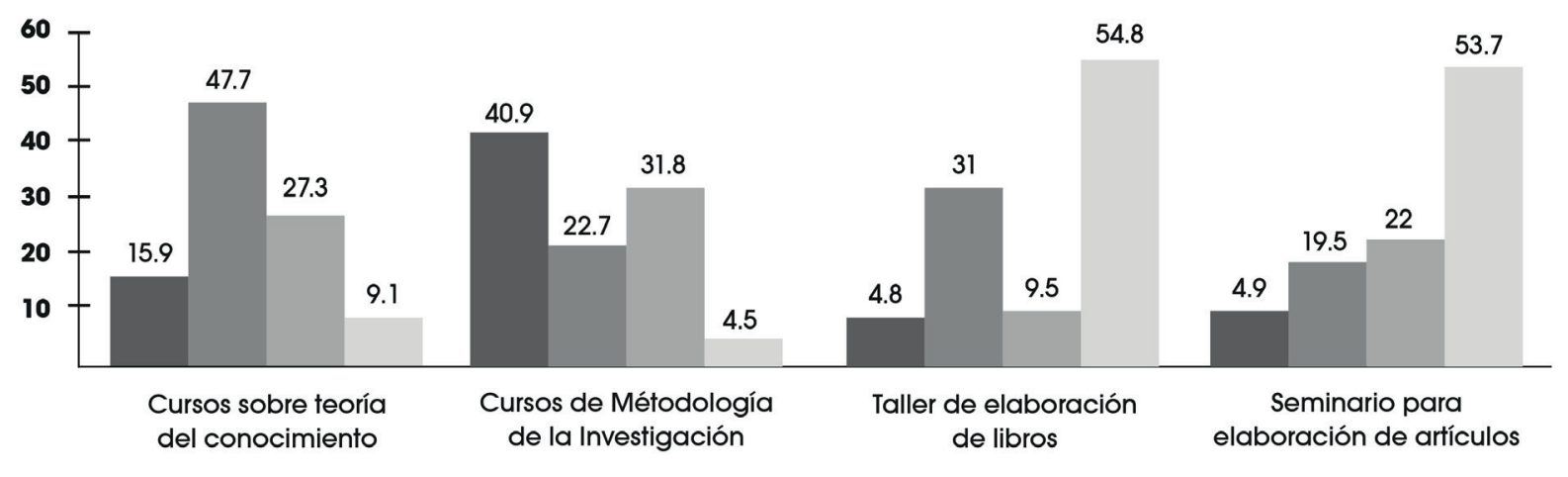

$\mathrm{Si}$, siempre los he tomado
A veces los he tomado
Muy pocas veces lo he tomado

No, nunca lo he impartido

Fuente: Cuestionarios aplicados a los docentes ENEO, FES Iztacala y FES Zaragoza, México, 2015.

68 
Gráfica 4. Asistencia de docentes a cursos de estadistica y busqueda bibliográfica

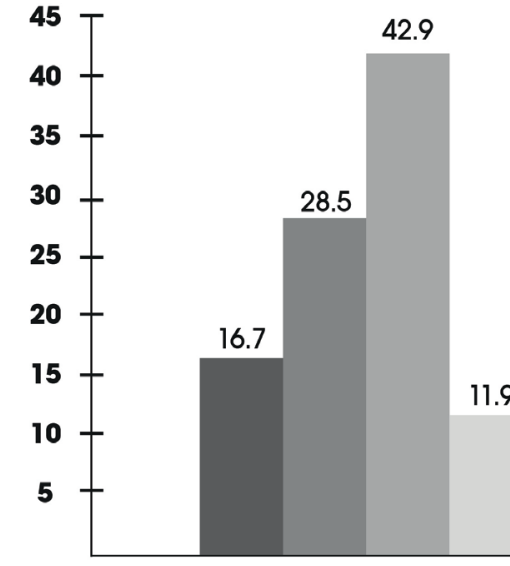

Taller de búsqueda bibliográfica

No, nunca lo he tomado
Muy pocas veces los he tomado
35.7

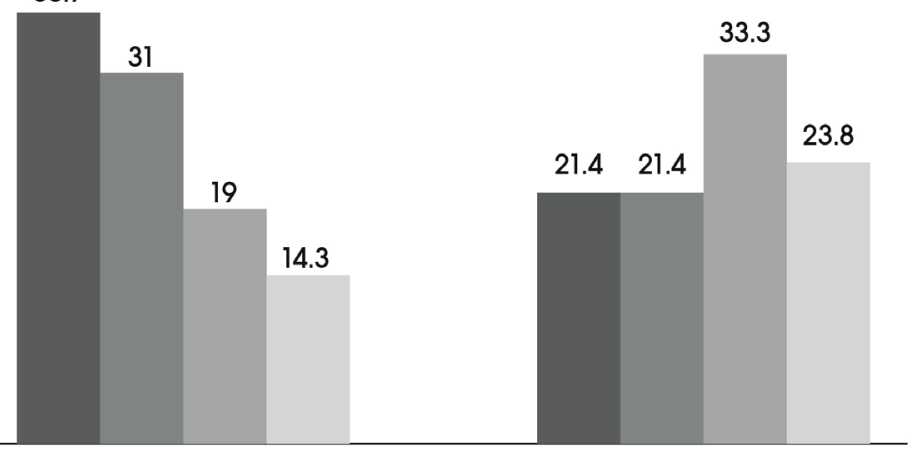

Cursos de estadística inferencial

Curso de estadística descriptiva

Gráfica 5. Asistencia a cursos de investigación cuali y cuantitativa

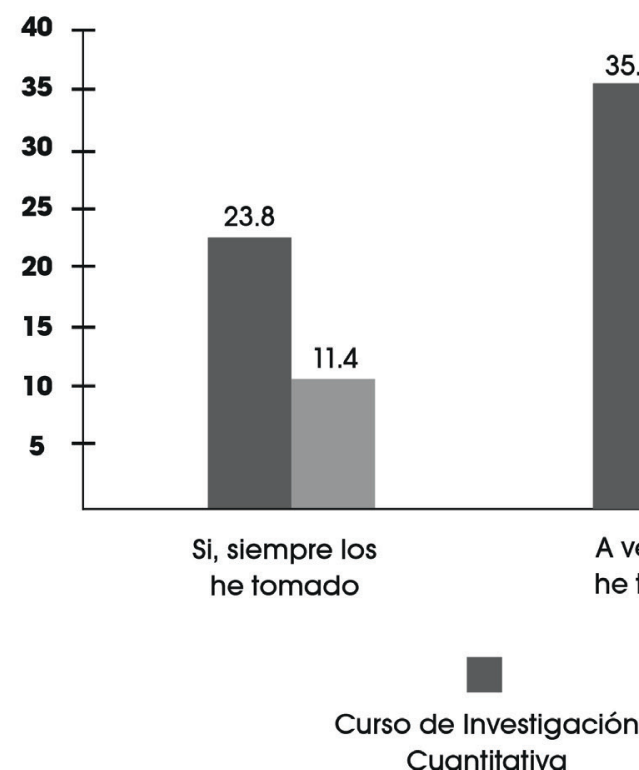

A veces los he tomado
Si, siempre los
he tomado

$$
\text { Cuantitativa }
$$

Curso de Investigación Cualitativa

Fuente: Cuestionarios aplicados a los docentes ENEO, FES Iztacala y FES Zaragoza, México, 2015. 


\section{REFERENCIAS BIBLIOGRÁFICAS}

1. Zarza Ma. D. Consolidación del sistema e investigación: Gestión de la investigación, calidad y productividad. Informe de la gestión 2011-2014. ENEO, 2015:1-100

2. Harrison L. Ray. A. Cianelli R. Rivera M.S y Urrutia M. Competencias en investigación para diferentes niveles de formación de enfermería. Ciencia y Enfermería, 2005: XI (1):7-59.

3. Miotto M.S. y Garzón N. Nuevos retos en el campo de la educación avanzada del personal de enfermería en América latina. Panamericana de Salud Pública, 1997; 2(1):57-56. Disponible en http://www.scielosp.org/pdf/ rpsp/v2n1/v2n1 a9.pdf. Consultado el día 1 de mayo del 2016

4. Mercado F.J. y Cols. Plataforma de Investigación en Salud: Una experiencia de formación participativa en una Universidad Mexicana. Enfermería Florianópolis, Oct- Dic. 2007; 16 (4): 753- 7561 Disponible en: hittp://www. escavador.com/pessoas/89781. Consultado el día 5 de Mayo del 2016

5. Cisneros M. Taller de escritura del artículo cientifico. Instituto de Investigaciones de la Amazonia Peruana, Julio. 2013; 14 (4): 1-6. Disponible en: http://www.ilap.org.pe/tallerarticulocientifico.pdf. Consultado el día 8 de Mayo del 2016

6. Faccia K. Estudio cualitativo y cuantitativo de la profesionalización de la enfermería desde una perspectiva antropológica. Argentina de Salud Pública, Diciembre. 2009; 1 (1): 12-17. Disponible en: http://www.saludinvestiga. org.ar/rasp/articulos/volumen1/RASP102-Faccia-ProfesionalizaciondeEnfer meriades.pdf. Consultado el día 15 de Mayo del 2016.

7. Palucci M. H. Producción científica de la enfermería brasileña: la búsqueda del impacto internacional. Latinoamericana de Enfermagen, Mayo- Junio, 2005: 13 (3): 289- 290. Disponible en: http://www.researchgate.net/profile/ Maria_Marziale/publication/266607807_PRODUCCIN_CIENTFICA_DE_ LA_ENFERMERA_BRASILEA_LA_BSQUEDA_DEL_IMPACTO_INTERNACIONAL/ links/54b4f5090cf28ebe92e4a19c.pdf. Consultado el día 9 de Mayo del 2016.

8. Lorenzini A y Dumet J. Publicaciones cientificas calificadas en la Enfermería Brasileña. Brasileña de Enfermería, julio-Agosto 2009; 62(4): 501-502. Disponible en: http://www.scielo.br/pdf/reben/v62n4/es_01.pdf. Consultado el día 9 de Mayo del 2016.
9. Díaz L.P. y Cols. Investigación de enfermería: una caracterización en dos publicaciones latinoamericanas. Avances en Enfermería, Jul- Dic. 2011; 29 (2) 285-293. Disponible en: http://www.scielo.org.co/pdf/aven/v29n2/v29n2a08 pdf. Consultado el día 11 de Mayo del 2016.

10. Instituto de Salud Carlos III de España. Líneas de Investigación de Enfermería en Europa. Instituto de Salud Carlos III de España, Septiembre. 2009: 22-26. Disponible en: http://www.revistaseden.org/files/art500_1.pdf Consultado el día 12 de Mayo del 2016.

11. Guerra M.D. y Cols. Investigación acción en la formación del profesorado universitario nóvel a través de la propuesta de una rúbrica. Educación Médica Superior, 2015; 29(2): 351-368. Disponible en: http://scielo.sld.cu/ scielo.php?scrip=sciarttext\&pid=\$0864-21412015000200014. Consultado el día 1 de Junio del 2016.

12. Liceras R.A. La investigación sobre formación del profesorado en didáctica de las Ciencias Sociales. Histodidactica, 2004; 8(1): 1-22. Disponible en: http://www.ub.edu/histodidactica/images/documentos/ pdf/investigacion formacion profesorado didactica ciencias sociales. pdf. Consultado el día 1 de Junio del 2016

13. Carro L. La formación del profesorado en investigación educativa: una visión crítica. Interuniversitaria de Formación del Profesorado. Diciembre, 2000 39: 15-32. Disponible en: http://maxconn.aufon.com/aufon/uploaded_files/ articulos/1223515897.pdf. Consultado el día 1 de junio del 2016

14. Guisasola J. y Cols. Formación continua del profesorado, investigación educativa e innovación en la enseñanza de las ciencias. Interuniversitaria de Formación del Profesorado. Agosto, 2007; 41: 207-222. Disponible en: http:// www.redalyc.org/pdf/274/27404173.pdf. Consultado el día 1 de junio 2016.

15. Romo A.E. y Cols. Gestión del conocimiento: estrategias para la formación de investigadores. Sinéctica. Universidad Jesuita de Guadalajara. Enero- Junio; 2012; 38: 1-20. Disponible en: http://www.scielo.org.mx/scielo php?script=sci_arttext\&pid=\$1665-109X2012000100007. Consultado el día de Junio del 2016. 